\title{
High-Strength Suture Tape Augmentation Improves Cyclic Gap Formation in Transosseous Patellar Tendon Repair: A Biomechanical Study
}

\author{
Heath P. Gould, M.D., David A. Weiner, M.D., Paul M. Tomaszewski, M.D., \\ Brent G. Parks, M.S., Pooyan Abbasi, M.S.M.E., and Allison L. Fillar, M.D.
}

\begin{abstract}
Purpose: To compare gap displacement at various intervals of cyclic testing and biomechanical load to failure of a Krackow patellar tendon repair augmented with high-strength suture tape versus the standard Krackow transosseous technique for inferior pole patellar tendon rupture. Methods: Twelve matched pairs of cadaveric knees were used (8 males and 4 females; mean age 79.6 years, range 57 to 96). An inferior pole patellar tendon rupture was simulated after random assignment of specimens in each pair to the standard or augmented Krackow technique. Each specimen was then repetitively cycled from $90^{\circ}$ to $5^{\circ}$ for 1,000 cycles. A differential variable reluctance transducer was used to measure gap displacement. After cyclic loading, load to failure was determined by pulling the tendon at a rate of 15 $\mathrm{mm} / \mathrm{s}$ until a sudden decrease in load occurred. Results: Compared with the control repair, specimens with augmented repair demonstrated significantly less displacement at all testing intervals up to 1,000 cycles $(P<.05)$. Two patellar tendons failed before the end of cyclic loading, and 4 specimens had inadequate tendon length for loading. Among the 18 remaining specimens, no significant difference in load to failure was observed between the experimental group $(\mathrm{n}=11)$ and the control group $(\mathrm{n}=7)(1,006.5 \pm 332.1$ versus $932.8 \pm 229.1 \mathrm{~N}$, respectively; $P=.567)$. Conclusions: Significantly greater gap displacement was observed in the standard Krackow repair group compared with the augmented Krackow group at all cyclic loading intervals. This suggests that the Krackow transosseous procedure augmented with high-strength suture tape is biomechanically viable for inferior pole patellar tendon repair. Clinical Relevance: This biomechanical study supports the use of high-strength suture tape augmentation of Krackow transosseous repair for inferior pole patellar tendon rupture.
\end{abstract}

\section{Introduction}

I solated patellar tendon rupture is a relatively uncommon injury, occurring most often as a result of acute trauma in males younger than 40 years. ${ }^{1}$ Surgical repair is the gold standard of treatment for patellar tendon rupture, with several operative techniques in

From the Department of Orthopaedic Surgery, MedStar Union Memorial Hospital, Baltimore, Maryland, U.S.A.

The authors report no conflicts of interest in the authorship and publication of this article. Full ICMJE author disclosure forms are available for this article

online, as supplementary material.

Received November 26, 2019; accepted May 2, 2020.

Address correspondence to Allison L. Fillar, M.D., clo Lyn Camire, MA, ELS, Editor, Department of Orthopaedic Surgery, MedStar Union Memorial Hospital, 3333 North Calvert St, Ste 400, Baltimore, MD 21218, U.S.A. E-mail:lyn.camire@medstar.net

Published by Elsevier on behalf of the Arthroscopy Association of North America. This is an open access article under the CC BY-NC-ND license (http://creativecommons.org/licenses/by-nc-nd/4.0/).

2666-061X/191415

https://doi.org/10.1016/j.asmr.2020.05.001 common use. Historically, the preferred method of patellar tendon repair has been the 4-strand locking Krackow transosseous technique. ${ }^{2}$ Several strategies for augmentation of this technique have been studied, including the use of cerclage wires, figure- 8 suture, and hamstring autograft. ${ }^{3-11}$ These augmented techniques have demonstrated superior biomechanical properties compared with the Krackow transosseous technique alone, which may facilitate advanced biomechanical stability to support accelerated rehabilitation protocols. $^{3-8}$

High-strength suture tape augmentation has been used successfully in a variety of orthopaedic procedures such as medial collateral ligament repair, ulnar collateral ligament repair, and lateral ankle ligament (Brostrom) repair to protect the primary surgical repair. ${ }^{12-15}$ Suture augmentation repair has also been described with the standard Krackow transosseous technique for patellar tendon repair. ${ }^{16}$ One biomechanical study found that the Krackow technique with high-strength suture tape augmentation was stronger than cerclage 
wire augmentation or the Krackow technique alone in repair of midsubstance patellar tendon rupture. ${ }^{17} \mathrm{~A}$ separate technique article described a patellar tendon repair method using a high-strength suture tape construct with unicortical buttons for fixation. ${ }^{16}$ However, the role of high-strength suture tape augmentation of the Krackow technique in the setting of inferior pole avulsion remains unclear. ${ }^{18}$

Midsubstance rupture is much less common than inferior pole rupture ${ }^{18}$ and also requires a different repair procedure and final construct. To repair a midsubstance rupture, a Krackow locking stitch is performed at both the proximal and distal limbs of the transected tendon and then hand-tied over the rupture site. In contrast, an inferior pole rupture requires a transosseous technique in which the Krackow locking stitch is performed only in the distal limb of the tendon, the sutures are passed through 3 drill holes in the patella, and the hand ties occur at the superior patellar pole. These differences require specific testing to assess whether internal brace augmentation is beneficial biomechanically for treatment of inferior pole patellar tendon defects.

The purpose of this study was to compare gap displacement at various intervals of cyclic testing and biomechanical load to failure of a Krackow patellar tendon repair augmented with high-strength suture tape versus the standard Krackow transosseous technique for inferior pole patellar tendon rupture. We hypothesized that the augmented Krackow construct would demonstrate superior biomechanical properties for inferior pole patellar tendon rupture compared with the standard repair alone.

\section{Methods}

This study was conducted at a single institution between August 2018 and October 2018. Twelve matched pairs of fresh frozen cadaveric knees were used ( 8 males and 4 females; mean age 79.6 years, range 57 to 96 ). Cadaveric specimens were thawed for $\geq 24$ hours. Specimens were assessed for study inclusion by the senior author (A.L.F.), a fellowship-trained orthopaedic sports medicine surgeon. Specimens were excluded if they had any evidence of significant pathological conditions of the patellar tendon. No specimens were excluded from the study based on these criteria.

Skin and subcutaneous tissues were removed from the specimens for ease of manipulation. ${ }^{19}$ The specimens in each pair were randomly assigned to undergo the Krackow transosseous repair or the internal braceaugmented Krackow repair. A biomechanical model of extensor mechanism cyclic loading originally described by Ravalin et al. ${ }^{3}$ and then refined by Krushinski et al. ${ }^{19}$ was used as the testing setup. The femur was fixed to an MTS Mini Bionix load frame (MTS Systems, Eden Prairie, MN) and oriented parallel to the floor using a custom-made clamp. The tibia was allowed to hang freely. The weight of an intact foot was simulated using a $2.3-\mathrm{kg}$ (5-lb) weight fixed $\sim 33 \mathrm{~cm}$ distal to the knee joint line using an external fixation device (Synthes, West Chester, PA). ${ }^{19,20}$ The quadriceps tendon was secured using a custom soft tissue clamp, which was attached to a heavy-gauge steel cable passed through a pulley from its origin at the servohydraulic actuator.

A patellar tendon rupture was simulated by transecting the tendon $\sim 3 \mathrm{~mm}$ distal to the inferior pole of the patella, leaving the remaining retinaculum intact. The control group was treated with a standard 4-strand transosseous patellar tendon repair. $^{21}$ The patellar tendon was secured with a locked running Krackow stitch consisting of two No. 2 high-tensile strength sutures (FiberWire; Arthrex, Naples, FL). Each suture was passed in an up-and-back fashion with 5 suture loops in each direction. The 4 suture strands were then passed through 3 transpatellar drill holes and tied at $45^{\circ}$ of flexion. The patellar tendon defect was then oversewn with 2-0 vicryl suture in the standard fashion (Fig lA).

High-strength suture tape augmentation (Internal Brace; Arthrex) was done using a modification of the Mackay technique (Fig 1B). ${ }^{20}$ Medial and lateral bone tunnels were drilled obliquely into the inferior aspect of the patella. These tunnels were placed in the central one-third of the patellar bone stock and oriented at $45^{\circ}$ with respect to the long axis of the patella. A single bone tunnel was then drilled at the
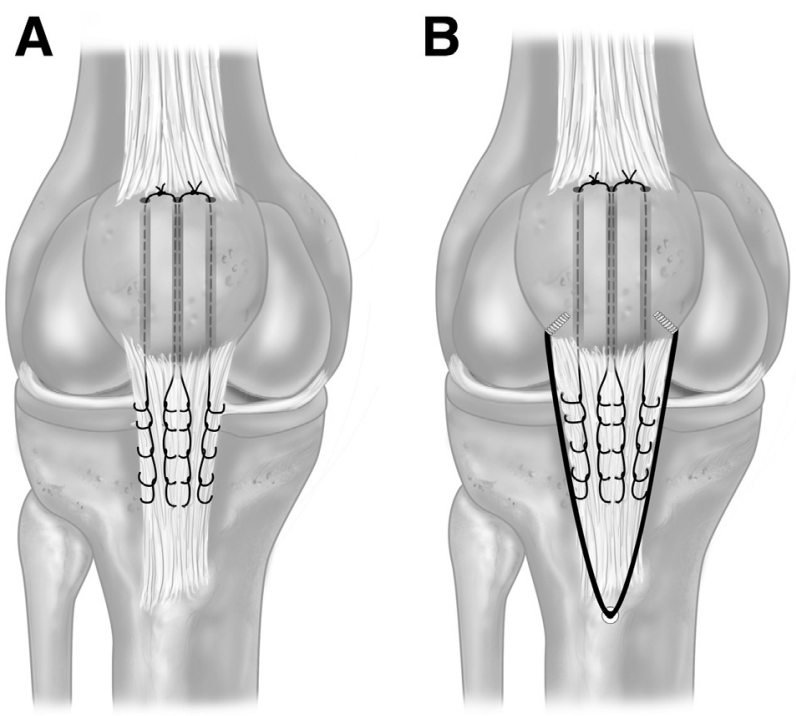

Fig 1. (A) Right knee Krackow transosseous repair without augmentation. (B) Right knee Krackow transosseous repair with high-strength suture tape augmentation. Suture anchors loaded with high-strength suture tape are placed medially and laterally at the inferior pole of the patella. The medial and lateral limbs of high-strength suture tape are secured distally with an additional anchor placed at the tibial tubercle. 
tibial tubercle perpendicular to the surface of the bone. A suture anchor (4.75-mm biocomposite knotless; SwiveLock; Arthrex) loaded with high-strength suture tape (FiberTape; Arthrex) was inserted into each proximal tunnel and tensioned until full range of motion was achieved without gapping at the site of the defect. Both limbs of Fibertape were then secured into the single distal bone tunnel at the tibial tubercle using an additional $4.75-\mathrm{mm}$ biocomposite knotless suture anchor.

After the repair was completed, a 9-mm stroke length differential variable reluctance transducer (DVRT) (Microstrain, Williston, VT) was secured to the anterior surface of the tendon spanning the repair site. The DVRT fixation points were augmented with a single simple-interrupted No. 2 high-tensile strength suture. Each specimen was then cycled from $90^{\circ}$ to $5^{\circ}$. Cyclic loading was stopped at $5^{\circ}$ rather than full extension to avoid the potential of placing nonanatomic stress on the repair by attempting to achieve a position beyond physiologic extension. ${ }^{19}$ The initial cycle was performed manually to obtain actuator displacement parameters, and therefore displacement values were not formally recorded for the initial cycle. Gap displacement was recorded at 2, 5, 20, 50, 100, 200, 500, and 1,000 cycles. After 1,000 cycles, the tendon was pulled at a rate of 15 $\mathrm{mm} / \mathrm{s}$. A sudden decrease in load on the DVRT was considered to represent load to failure.

Power analysis using a prior study that assessed patellar tendon gap formation between a pretension group and control group at 1,000 cycles ${ }^{19}$ determined that 12 specimens per group would provide $95 \%$ power to detect a statistically significant difference $(\alpha<0.05)$. Gap displacement was compared between groups at all cycle intervals using a paired $t$ test, and load to failure was compared using an unpaired $t$ test.

\section{Results}

Compared with controls, the augmented patellar tendon repair group demonstrated significantly less gap formation at all testing intervals (Table 1). Two patellar tendons in the control group failed during cyclic testing. Four additional patellar tendons from different cadaveric donors (3 control, l experimental) did not have adequate quadriceps tendon length for failure loading. No significant difference in load to failure was observed between the control group and the experimental group $(932.8 \pm 229.1$ and $1,006.5 \pm 332.1$, respectively; $P=$ .567). In the control group, 6 specimens failed by knot breakage at the superior pole of the patella, and 1 specimen failed by suture tearing at the bone-patellar tendon interface. In the augmented group, 6 specimens failed by knot breakage at the superior pole of the patella, 3 specimens failed by suture anchor pullout, 1 specimen failed by suture tearing at the bone-patellar
Table 1. Mean Gap Displacement (mm)

\begin{tabular}{lccc}
\hline & \multicolumn{2}{c}{ Krackow Transosseous Repair } & \\
\cline { 2 - 3 } Cycles & Standard & Augmented & $P$ Value \\
\hline 1 & NA & NA & \\
2 & $0.89 \pm 0.91$ & $0.18 \pm 0.27$ & $.016^{*}$ \\
5 & $1.63 \pm 1.41$ & $0.44 \pm 0.47$ & $.011^{*}$ \\
10 & $2.04 \pm 1.58$ & $0.61 \pm 0.57$ & $.011^{*}$ \\
20 & $2.36 \pm 1.56$ & $0.90 \pm 0.80$ & $.017^{*}$ \\
50 & $2.90 \pm 1.61$ & $1.27 \pm 1.08$ & $.014^{*}$ \\
100 & $3.22 \pm 1.56$ & $1.56 \pm 1.31$ & $.016^{*}$ \\
200 & $3.62 \pm 1.47$ & $1.66 \pm 1.42$ & $.005^{*}$ \\
500 & $4.22 \pm 1.38$ & $2.00 \pm 1.75$ & $.012^{*}$ \\
1,000 & $4.45 \pm 1.33$ & $2.27 \pm 1.83$ & $.014^{*}$ \\
\hline
\end{tabular}

Data are presented as mean gap displacement \pm standard deviation, in millimeters. NA, not applicable.

${ }^{*} P$ value significant at $\alpha<.05$.

tendon interface, and 1 specimen failed by tearing of the internal brace at the bone-patellar tendon interface.

\section{Discussion}

In this cadaveric study, significantly less displacement with cyclic loading was observed in the Krackow repair augmented with high-strength suture tape compared with standard Krackow transosseous repair. As has been shown previously for midsubstance patellar tendon rupture, ${ }^{17}$ these findings suggest that Krackow transosseous repair augmented with high-strength suture tape is biomechanically viable for inferior pole patellar tendon repair that does not require autograft harvest or implantation of metallic hardware. This additional protection against early gapping may have important clinical applications, potentially allowing for earlier postoperative motion and accelerated rehabilitation protocols that could ultimately lead to a faster return to play in athletes.

Our results are consistent with those of previous studies that have shown significantly improved gap formation with other augmentation strategies for inferior pole patellar tendon repair. ${ }^{3,5,7}$ At 250 cycles, Ravalin et al. ${ }^{3}$ reported significantly greater gap formation with the standard Krackow transosseous technique $(7.3 \pm 0.5 \mathrm{~mm})$ compared with augmentation with either Ethibond suture $(4.9 \pm 0.5 \mathrm{~mm})$ or 2.0 DallMiles cables $(3.5 \pm 0.8 \mathrm{~mm})$. Similarly, Black et al. ${ }^{5}$ reported improved gap formation at 250 cycles $(3.55 \pm 0.56 \mathrm{~mm})$ with a figure- 8 suture augmentation compared with the standard Krackow transosseous technique $(5.96 \pm 0.86 \mathrm{~mm})$. These values compare favorably to our internal brace-augmented construct, which demonstrated a mean gap formation of $1.66 \pm 1.42 \mathrm{~mm}$ at 200 cycles and $2.00 \pm 0.56 \mathrm{~mm}$ at 500 cycles. Mihalko et al. ${ }^{7}$ also reported superior results in gap displacement with hamstring augmentation $(7.2 \pm 0.9 \mathrm{~mm})$ compared with the Krackow transosseous repair alone $(13.2 \pm 1.9 \mathrm{~mm})$, although 
the displacement values presented for both the augmented and control repair groups at 250 cycles were much higher than the values reported in our study, as well as the other 2 prior studies. 3.5

Our findings also support those of a previous study by Rothfeld et al. ${ }^{17}$ that examined midsubstance patellar tendon repair and reported less gap formation at 250 cycles with their internal brace-augmented construct $(2.1 \pm 1.2 \mathrm{~mm})$ compared with the Krackow transosseous repair alone $(4.2 \pm 2.3 \mathrm{~mm})$. Our quantitative results are similar to those of Rothfeld et al. ${ }^{17}$ and are likely to confer a clinically significant benefit, given that prior biomechanical studies have defined clinical failure as 3- to 5-mm gap formation. ${ }^{3,19}$ Anecdotally, our highstrength suture tape augmentation procedure used fewer materials compared with the previous study, in which the investigators used 4 FiberTape-laden suture anchors ( 2 patellar and 2 tibial) to produce a "box-and$\mathrm{x}^{\prime \prime}$ configuration. We created a V-shaped configuration that required only 3 anchors ( 2 patellar and 1 tibial), potentially decreasing the cost and operative time associated with high-strength suture tape-augmented patellar tendon repair. Our findings suggest that this configuration may warrant further clinical study.

\section{Limitations}

Several limitations to our study design can be identified. Because the study was biomechanical, we could not account for the effects of tissue healing or specific stresses related to rehabilitation or daily activities. Six of the 24 specimens $(25 \%)$ could not be loaded to failure because the tendon was too short or they had already failed. Our simulation of a patellar tendon rupture by cleanly transecting the tendon with a scalpel blade is not representative of the more ragged tendon morphology that is often encountered clinically. Although this methodology for simulating tendon rupture is consistent with previous biomechanical studies, our results may not be directly generalizable to clinical practice in the absence of additional in vivo data. Also, we performed the transection just distal to the inferior patellar pole, rather than at the midsubstance of the patellar tendon. Although this allowed us to validate the application of an internal brace for the most commonly encountered variant of patellar tendon ruptures, it does not allow direct comparison with previous reports.

\section{Conclusion}

Significantly greater gap displacement was observed in the standard Krackow repair group compared with the augmented Krackow group at all cyclic loading intervals. This suggests that the Krackow transosseous procedure augmented with high-strength suture tape is biomechanically viable for inferior pole patellar tendon repair.

\section{Acknowledgments}

This study was supported by departmental research funds. We thank Lyn Camire, MA, ELS, of our department for editorial assistance and the Department of Orthopaedic Surgery for study funding.

\section{References}

1. Enad JG. Patellar tendon ruptures. South Med J 1999;92: 563-566.

2. Ong BC, Sherman O. Acute patellar tendon rupture: A new surgical technique. Arthroscopy 2000;16:869-870.

3. Ravalin RV, Mazzocca AD, Grady-Benson JC, Nissen CW, Adams DJ. Biomechanical comparison of patellar tendon repairs in a cadaver model: An evaluation of gap formation at the repair site with cyclic loading. Am J Sports Med 2002;30:469-473.

4. Bouget P, Breque C, Beranger JS, Faure JP, Khiami F, Vendeuvre T. Biomechanical cadaveric comparison of patellar ligament suture protected by a steel cable versus a synthetic cable. J Exp Orthop 2017;4:9.

5. Black JC, Ricci WM, Gardner MJ, et al. Novel augmentation technique for patellar tendon repair improves strength and decreases gap formation: A cadaveric study. Clin Orthop Relat Res 2016;474:2611-2618.

6. Meyer Z, Ricci WM. Knee extensor mechanism repairs: Standard suture repair and novel augmentation technique. J Orthop Trauma 2016;30:S30-S31 (suppl 2).

7. Mihalko WM, Vance M, Fineberg MJ. Patellar tendon repair with hamstring autograft: A cadaveric analysis. Clin Biomech (Bristol, Avon) 2010;25:348-351.

8. Van der Bracht H, Verdonk R, Stuyts B. Augmentation of a patellar tendon repair with an autologous semitendinosus graft. Acta Orthop Belg 2009;75:417-419.

9. Wuller TG, Jansson KA, Bruner BW. Augmentation of the patellar ligament with a bone-patellar tendon-bone inlay allograft. Arthroscopy 1998; 14:89-93.

10. Woodmass JM, Johnson JD, Wu IT, Krych AJ, Stuart MJ. Patellar tendon repair with ipsilateral semitendinosus autograft augmentation. Arthrose Tech 2017;6: e2177-e2181.

11. Espregueira-Mendes J, Andrade R, Michael M, et al. Augmentation of patellar tendon repair with autologous semitendinosus Graft-Porto technique. Arthrosc Tech 2017; 6:e2271-e2276.

12. Lubowitz JH, MacKay G, Gilmer B. Knee medial collateral ligament and posteromedial corner anatomic repair with internal bracing. Arthrosc Tech 2014;3:e505-e508.

13. Dugas JR, Walters BL, Beason DP, Fleisig GS, Chronister JE. Biomechanical comparison of ulnar collateral ligament repair with internal bracing versus modified jobe reconstruction. Am J Sports Med 2016;44: 735-741.

14. Jones CM, Beason DP, Dugas JR. Ulnar collateral ligament reconstruction versus repair with internal bracing: Comparison of cyclic fatigue mechanics. Orthop J Sports Med 2018;6:2325967118755991.

15. Yoo JSY EA. Clinical results of an arthroscopic modified Brostrom operation with and without an internal brace. J Orthop Traumatol 2016;17:353-360. 
16. Sanchez G, Ferrari MB, Sanchez A, et al. Proximal patellar tendon repair: Internal brace technique with unicortical buttons and suture tape. Arthrosc Tech 2017;6: e491-e497.

17. Rothfeld A, Pawlak A, Liebler SAH, Morris M, Paci JM. Patellar tendon repair augmentation with a knotless suture anchor internal brace: A biomechanical cadaveric study. Am J Sports Med 2018;46: $1199-1204$.

18. Yousef MAA. Combined avulsion fracture of the tibial tubercle and patellar tendon rupture in pediatric population: Case series and review of literature. Eur J Orthop Surg Traumatol 2018;28:317-323.
19. Krushinski EM, Parks BG, Hinton RY. Gap formation in transpatellar tendon repair: Pretensioning Krackow sutures versus standard repair in a cadaver model. Am J Sports Med 2010;38:171-175.

20. Mackay GM, Blyth MJ, Anthony I, Hopper GP, Ribbans WJ. A review of ligament augmentation with the InternalBrace: The surgical principle is described for the lateral ankle ligament and ACL repair in particular, and a comprehensive review of other surgical applications and techniques is presented. Surg Technol Int 2015;26:239-255.

21. Marder RA, Timmerman LA. Primary repair of patellar tendon rupture without augmentation. Am J Sports Med 1999;27:304-307. 